EUROPEAN ORGANIZATION FOR NUCLEAR RESEARCH

European Laboratory for Particle Physics

arge Hadron Collider Project

LHC Project Report 512

\title{
QUENCH PROPAGATION \\ IN THE SUPERCONDUCTING 6 KA FLEXIBLE BUSBARS OF THE LHC
}

R. Herzog, M. Calvi, F. Sonnemann and J.M. Pelegrin-Carcelen

\begin{abstract}
Flexible superconducting cables with currents up to $6 \mathrm{kA}$ will be used to power magnets individually in the insertion regions of the LHC. In case of a quench, the currents in these circuits will decay very fast (with time constants of about $200 \mathrm{~ms}$ ) such that relatively small copper cross sections are sufficient for these busbars.

Quench propagation experiments on a prototype cable and corresponding simulations led to a detailed understanding of the quench behavior of these busbars and to recommendations for the design and application of the cable. Simulations of the quench process in a multi-strand conductor led to a detailed understanding of the way current crosses from superconducting to pure copper strands and how this affects the quench propagation velocity.

At nominal current $(6 \mathrm{kA})$, the quench propagation velocities are high $(10 \mathrm{~m} / \mathrm{s})$ and the hot spot temperature increases rapidly. In this situation, timely quench detection and energy extraction (current reduction) are vital to prevent damage of circuit components.
\end{abstract}

LHC Division

Presented at the 2001 Cryogenic Engineering Conference and International Cryogenic Materials Conference CEC/ICMC 2001

16-20 July 2001, Madison, Wisconsin, USA

Administrative Secretariat

LHC Division

CERN

CH - 1211 Geneva 23

Switzerland

Geneva, 19 October 2001 


\title{
QUENCH PROPAGATION IN THE SUPERCON- DUCTING 6 kA FLEXIBLE BUSBARS OF THE LHC
}

\author{
R. Herzog, M. Calvi, F. Sonnemann and J. M. Pelegrin-Carcelen \\ CERN, LHC Division, 1211 Geneva 23, Switzerland
}

\begin{abstract}
Flexible superconducting cables with currents up to $6 \mathrm{kA}$ will be used to power magnets individually in the insertion regions of the LHC. In case of a quench, the currents in these circuits will decay very fast (with time constants of about $200 \mathrm{~ms}$ ) such that relatively small copper cross sections are sufficient for these busbars.

Quench propagation experiments on a prototype cable and corresponding simulations led to a detailed understanding of the quench behavior of these busbars and to recommendations for the design and application of the cable. Simulations of the quench process in a multi-strand conductor led to a detailed understanding of the way current crosses from superconducting to pure copper strands and how this affects the quench propagation velocity.

At nominal current $(6 \mathrm{kA})$, the quench propagation velocities are high $(10 \mathrm{~m} / \mathrm{s})$ and the hot spot temperature increases rapidly. In this situation, timely quench detection and energy extraction (current reduction) are vital to prevent damage of circuit components.
\end{abstract}

\section{INTRODUCTION}

At the ends of the eight regular arcs of the LHC, in the so called matching sections and insertion regions, a number of special quadrupoles (MQMs) and dipoles (MBRs) need to be individually powered with currents up to $6 \mathrm{kA}$. With the successful adoption of the concept of superconducting $600 \mathrm{~A}$ cables being pulled into tubes during the installation of the magnets in the tunnel [1] and similar topological needs, the development of $6 \mathrm{kA}$ flexible cables was envisaged. To study the feasibility of a multiconductor cable with such a high current rating, a series of experiments were conducted on a prototype built by a commercial cable manufacturer. The two main concerns addressed by the tests were the robustness of the cable to exclude any quenches triggered by conductor movement inside the cable and the temperature development once a quench was triggered by some outside effect (e.g. by beam losses). A program developed for the simulation of quench processes [2] greatly helped in the understanding of the quench details and in the evaluation of the quench protection system. 
TABLE 1. Required cable properties

\begin{tabular}{ll|ll}
\hline Parameter & Value & Parameter & Value \\
\hline Number of conductors & 6 & Copper cross section of one conductor & $9.6 \mathrm{~mm}^{2}$ \\
Maximum current per conductor at $1.9 \mathrm{~K}$ & $6 \mathrm{kA}$ & Minimum RRR of copper & 100 \\
Min. critical current at $1.9 \mathrm{~K}$ and $50 \mathrm{mT}$ & $11 \mathrm{kA}$ & Insulation withstand voltage & $3 \mathrm{kV}$ \\
Minimum bending radius of cable & $200 \mathrm{~mm}$ & Radiation dose the cable has to withstand & $60 \mathrm{kGy}$ \\
\hline
\end{tabular}

\section{DESIGN OF THE PROTOTYPE CABLE}

The design of the prototype cable, shown in FIG 1, is based on the requirements of the LHC and on the experience gained with a multipolar $600 \mathrm{~A}$ cable [1]. The availability of a large quantity of superconducting strand at the Institute of High Energy Physics in Protvino, Russia, which was well adapted to the needs of the cable, led to a collaboration between IHEP and CERN.

The cable needs to be flexible for the installation in a tube welded to the cold masses of large LHC cryo-magnets. Bending the cable to a radius of $200 \mathrm{~mm}$ must be an easy manual operation and well within the elastic limit of deformation. On the other hand the cable needs to be designed in such a way that the large mutual Lorentz forces (up to $1800 \mathrm{~N} / \mathrm{m}$ ) of the conductors inside are taken up by the structure and do not lead to movements causing quenches. The cable will operate immersed in superfluid helium at $1.9 \mathrm{~K}$.

If a quench is detected in a magnet powered by the cable or in the cable itself, quench heaters are fired in the corresponding magnet to initiate a rapid energy dissipation and current reduction. With the short times of detection $(<100 \mathrm{~ms})$, verification $(\sim 20 \mathrm{~ms})$, heater delay $(\sim 30 \mathrm{~ms})$ and current decay $(\sim 150 \mathrm{~ms}$ at $6 \mathrm{kA})$, which were calculated with adiabatic assumptions, a copper stabilization of about $8 \mathrm{~mm}^{2}$ is sufficient to prevent damage of the busbar. To obtain some margin, the prototype was therefore built with a copper cross-section of $9.6 \mathrm{~mm}^{2}$. The assessment of this choice was one of the aims of the simulations and experiments on the prototype cable, which are described below.

A single conductor is built of 11 superconducting strands with a copper to NbTi ratio of 1.38 and a diameter of $0.85 \mathrm{~mm}, 9$ copper strands with the same diameter and 1 central copper strand of $1.1 \mathrm{~mm}$ diameter in the center. The inner layer is made of 7 copper strands and has a pitch length of $25 \mathrm{~mm}$. All superconducting strands are in the outer layer to ensure a uniform current distribution among them [3].

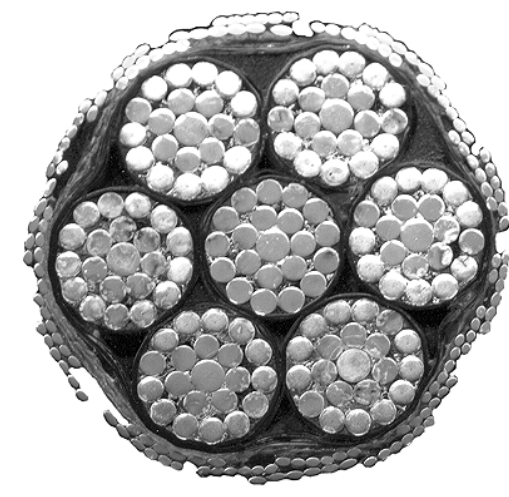

FIGURE 1. Cross section of the $6 \mathrm{kA}$ prototype cable. The 11 lighter strands in the outer layer of each conductor are the superconducting ones. Conductor diameter: $5 \mathrm{~mm}$, Cable diameter: $16.7 \mathrm{~mm}$ With two copper strands they form the outer layer with a pitch length of $44 \mathrm{~mm}$. The two layers are wound in opposite directions. The $6 \mathrm{kA}$ conductor is insulated with two layers of $50 \mu \mathrm{m}$ polyimide tape ( $48 \%$ overlap) to achieve a total thickness of $0.2 \mathrm{~mm}$.

Six conductors are laid around a central conductor of the same dimensions, which contains only copper strands, to form the cable. On the outside, it is protected against impacts by a polyester bedding tape and a stainless steel braid. The total diameter is $16.7 \mathrm{~mm}$. TABLE 1 summarizes some cable properties.

\section{ELECTRICAL MEASUREMENTS AT 1.9 K}

The main purpose of the experiments conducted on the prototype cable was to show that a flexible and 
compact multipolar cable could carry currents up to $6 \mathrm{kA}$ without quenching. In addition it was intended to investigate the quench behavior of the cable at all current levels and measure the characteristic currents $I_{0}$ and $I_{1}$ and the quench propagation velocity $v_{\mathrm{q}}(I)$.

\section{Experimental Set-Up}

The helium cryostat in which the experiments were carried out provided only one pair of current leads rated above $2 \mathrm{kA}$. Four adjacent conductors inside the cable were thus connected in series as shown in FIG 3 to generate the largest possible Lorentz forces on the two external conductors. To equip a $6 \mathrm{~m}$ long section of the cable with 24 voltage taps and two small heaters for provoking quenches, the stainless steel braid was removed from several areas about $8 \times 8 \mathrm{~mm}^{2}$ large. After removing the insulation, wires were soldered to the conductors. Once the cable was fully instrumented, it was led through the aperture of a MCBX prototype magnet, which can generate a dipole field of up to $3.5 \mathrm{~T}$, and wound around four steel rods as shown in FIG 2. The lower ends of the rods additionally held an insulated steel sheet onto which $5 \mathrm{~m}$ of $600 \mathrm{~A}$ wire were tied for quench propagation experiments through feedthroughs [4]. A 32-channel data logger recorded the voltages between adjacent taps at frequencies up to $10 \mathrm{kHz}$. A $20 \mathrm{kA}$ switch mode power converter supplied the currents for the $6 \mathrm{kA}$ cable, and by discharging a large capacitor into either of the two heaters quenches could be triggered.

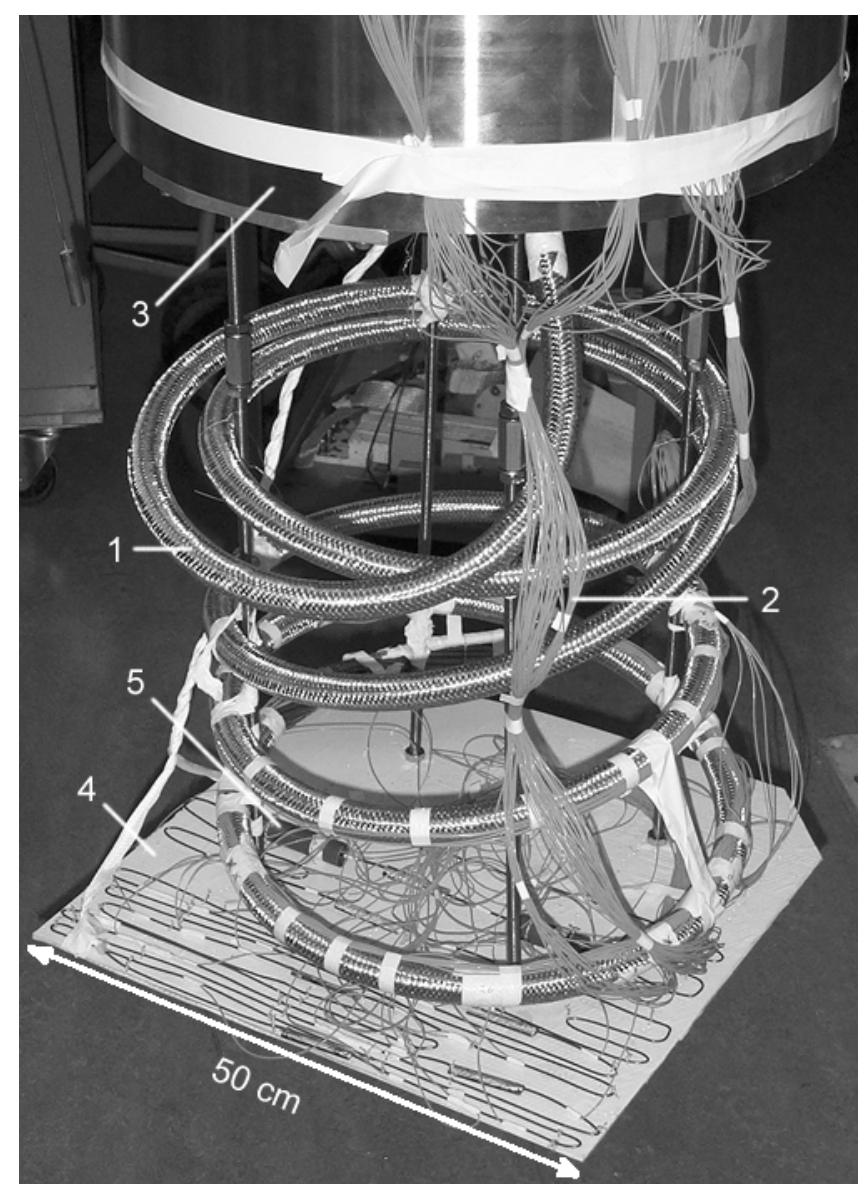

FIGURE 2. Experimental setup for quench propagation measurements. $16 \times 6 \mathrm{kA}$ cable, 2 voltage tap wires, 3 MCBX prototype dipole, 4 insulated steel sheet with 600 A busbar wire mounted, 5 'feedthrough' on 600 A wire.

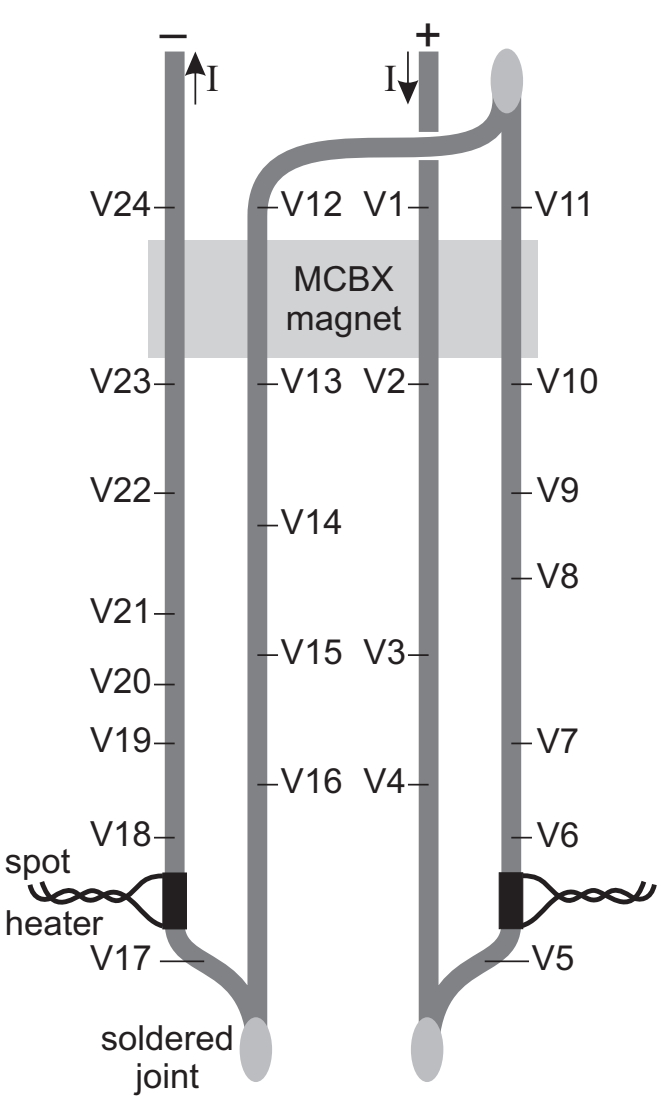

FIGURE 3. Schematic diagram of the $6 \mathrm{kA}$ test circuit with 24 voltage taps, two spot heaters and three wire joints. 


\section{Operation At Nominal Conditions}

During current ramps in $1 \mathrm{kA}$ steps up to $6 \mathrm{kA}$ with a rate of $20 \mathrm{~A} / \mathrm{s}$, the voltages on all channels were recorded with a sample frequency of $10 \mathrm{kHz}$ to see if movements of conductor sections possibly caused any voltage spikes. With the noise level of the experiment and recording system at $5 \mathrm{mV}$, no spikes could be detected however. Even with a field of $1.5 \mathrm{~T}$ transversal to the cable in the MCBX magnet, no spikes were observed, indicating that the cable structure firmly holds the conductors in place. Ramps up to $10 \mathrm{kA}$ eventually resulted in conductor movements that also led to quenches, but careful analysis of the data and inspection of the cable after it had been taken out of the cryostat clearly showed that these movements occurred at the lower end of the installed cable section, where the steel braid had been removed, the joints been soldered and the conductors apparently not been tied down sufficiently. Later tests with a different set-up revealed that the critical current of the conductor is not reached at $14 \mathrm{kA}$ and that it even withstood heater triggered quenches at these levels for $120 \mathrm{~ms}$ between quench onset and current turn off.

\section{Quench Performance}

With constant currents at many levels between $800 \mathrm{~A}$ and $7 \mathrm{kA}$ in the cable, the two small heaters were used to trigger quenches. The expansion of the normal zone was subsequently registered by the appearance of resistance and hence voltage in the sections between successive voltage taps. FIG 4 shows typical voltage traces recorded at $2 \mathrm{kA}$. The voltage rises sharply from zero when the normal zone enters a conductor section monitored my two voltage taps, increases more or less linearly while the front line between normal and superconducting zones propagates from one tap to the other and rises much more slowly (or even settles if $I<I_{1}$ ) once the front line has passed the second tap. At that moment a small peak appears, which has not been observed in single strand busbars [4]. The suspicion that it might have something to do with the way current sharing between the superconducting and the copper strands occurs could be confirmed by simulations described below.

From the passage of the border between normal and superconducting zones at successive voltage taps the quench propagation velocity was calculated. It is shown in FIG 5 as a

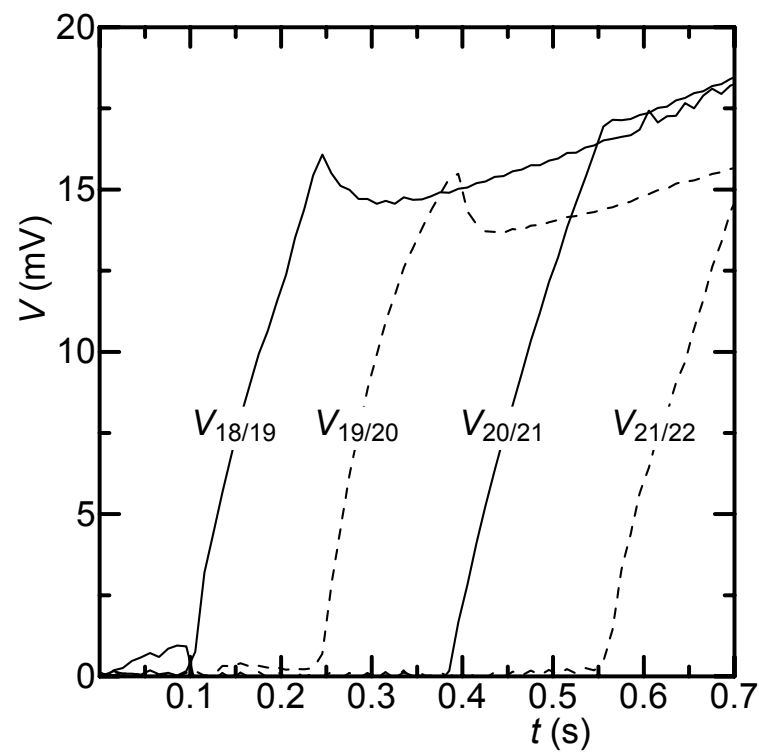

FIGURE 4. Typical voltages recorded between neighboring taps over time. The local voltage peaks in one channel occur when the quench front passes into the next (e.g. $V_{18 / 19}$ peak at first rise of $V_{19 / 20}$ ).

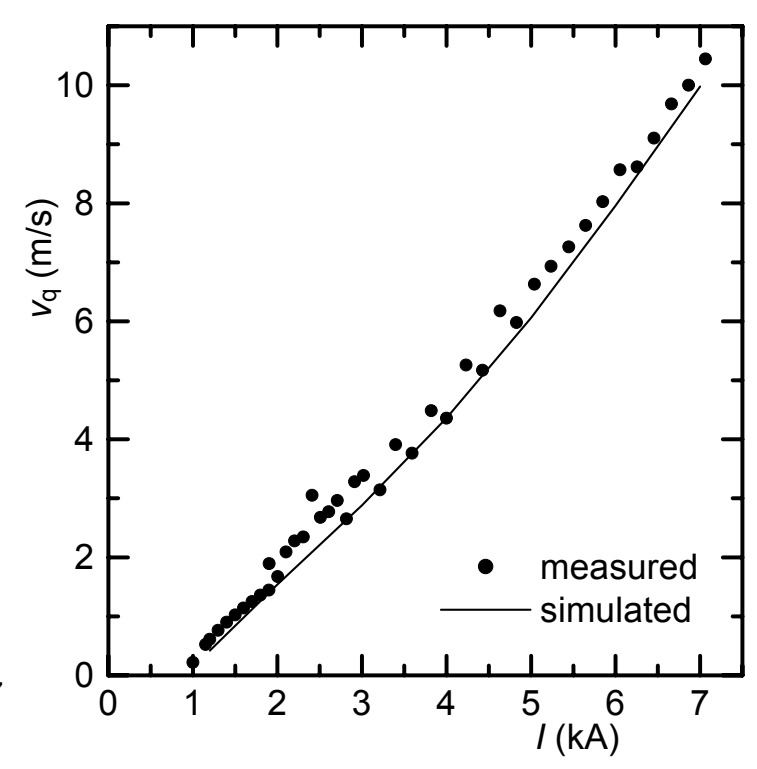

FIGURE 5. Quench propagation velocity as a function of current with the optimized parameters $A_{\mathrm{He}}=1.4 \mathrm{~mm}^{2}$ and $G_{\mathrm{c}}=10^{7}(\Omega \mathrm{m})^{-1}$. 
function of the current in the conductor. The current where the velocity is zero $\left(I_{0}\right)$ is about $960 \mathrm{~A}$. The determination of $I_{1}$, the current above which no stable equilibrium between heating and cooling in the resistive zone exists, was not as clear as with the single strand busbars described in [4]. The probable reason is the higher heat generation at the equilibrium point, because of the larger circumference, which leads to a change of the conductor's helium environment inside the cable. No real equilibrium could therefore be found; after several seconds the temperature of the conductor was increasing ever more rapidly. Another sign for the heating up of the helium environment was the provocation of a quench in a neighboring conductor. Furthermore, the quantity of helium in contact with the strands inside the insulation of the conductor adds heat capacity to the heat capacity of the metal. Per unit volume and at $9 \mathrm{~K}$ it is actually 2 times larger than the one of copper. Nevertheless, at $1500 \mathrm{~A}$ the hot-spot temperature remained nearly constant for several seconds during the experiments.

\section{Quench Propagation Simulations}

The finite difference model for the simulation of quench phenomena described in [4] had to be extended to consider the presence of liquid helium inside the conductor insulation and to take into account that half of the strands are made of pure copper. The first extension had already been made to simulate the quench behavior of magnet coils [5] and could be readily applied here.

The simplest electrical model of superconducting and pure copper strands laid together into a single conductor and therefore touching each other all along is shown in FIG 6. All superconducting strands are grouped into the upper chain of resistors, all copper ones into the lower chain. Each finite element of the upper chain is connected to the adjacent element of the lower chain by a resistor acting as the contact resistance between the two strand types. For the simulation of a propagating quench the same temperature profile $T(z, t)$ was assumed for both resistor chains, but after each time increment, the currents in the resistor network were recalculated following Kirchhoff's laws. From the current distribution and the resistances, which are functions of $T(z, t)$, the energy dissipation in each element was computed and after considering thermal flows a new temperature profile could be determined.

FIG 7. shows the time development of some important variables calculated with the parameters of the prototype cable. Along most of the normal zone, the current is shared among the superconducting and the copper strands according to their copper cross-section (about 3:5), whereas all the current is flowing in the superconducting strands in the parts of the conductor at $1.9 \mathrm{~K}$. In the vicinity of the border between the two zones, just where the temperature reaches $T_{\mathrm{c}}$, the current crosses from the superconducting to the copper strands at the 'upstream' boundary (plus pole side) of the normal zone and returns at the 'downstream' boundary. The length of these sections with transverse current increases with the contact resistance per unit length of conductor. For the simulations, the value of the contact resistance was assumed location and temperature independent, and it was chosen such that

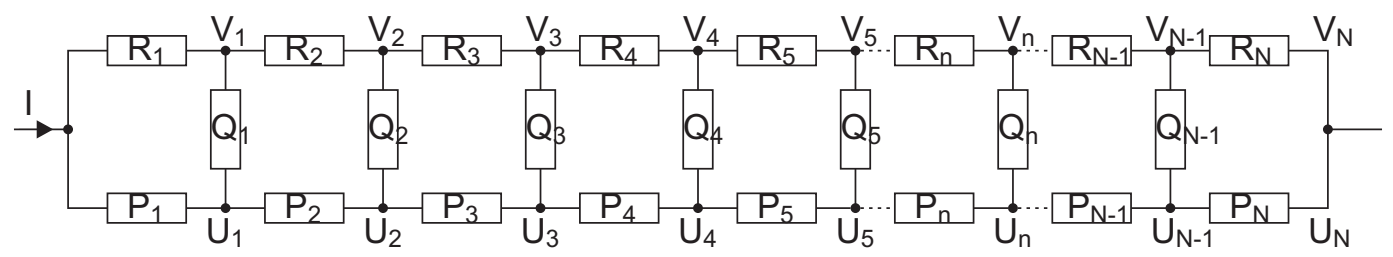

FIGURE 6. Resistor network to model superconducting and pure copper strands in parallel and touching. The $R_{\mathrm{n}}$ are resistances of the sc. elements, the $P_{\mathrm{n}}$ the ones of the copper elements. Both are the $T$ dependent. The contact resistances $Q_{\mathrm{n}}$ were assumed $T$ independent and all equal. The contact conductivity per unit length of conductor $G_{\mathrm{c}}$ corresponds to $\left(Q_{\mathrm{n}} \cdot \Delta z\right)^{-1}$, with $\Delta z$ the length of the conductor element. 
the experimentally recorded voltages were reproduced as closely as possible. It is noteworthy that transverse currents and currents in the copper strands appear in regions where $T$ is well below $T_{\mathrm{c}}$. Ohmic heating therefore arises already 'ahead' of the expanding normal zone, which significantly increases the quench propagation velocities in comparison with a single strand of the same copper cross section (by about a factor of 2 for the best fitting contact conductivity $G_{\mathrm{c}}=10^{7}(\Omega \mathrm{m})^{-1}$ ).

The potential of the copper strands is different from the potential of the superconducting strands only in the regions with a transverse current. FIG 8 shows the two potentials along the conductor for a given moment $\left(t_{0}\right)$. Since the outer strands of the conductor are mostly superconducting, soldered on voltage taps are likely to record their potential. After a short initial phase at the start of a quench, the front line of a normal zone advances in a way where the detailed shapes of the variables around it remain constant and move with it. It is thus possible to reconstruct the voltage between two neighboring taps by following the advance of the superconducting strands' potential (see FIG 8). At $t_{1}$, the quench enters the section between taps 1 and 2, the first voltage arises and it increases
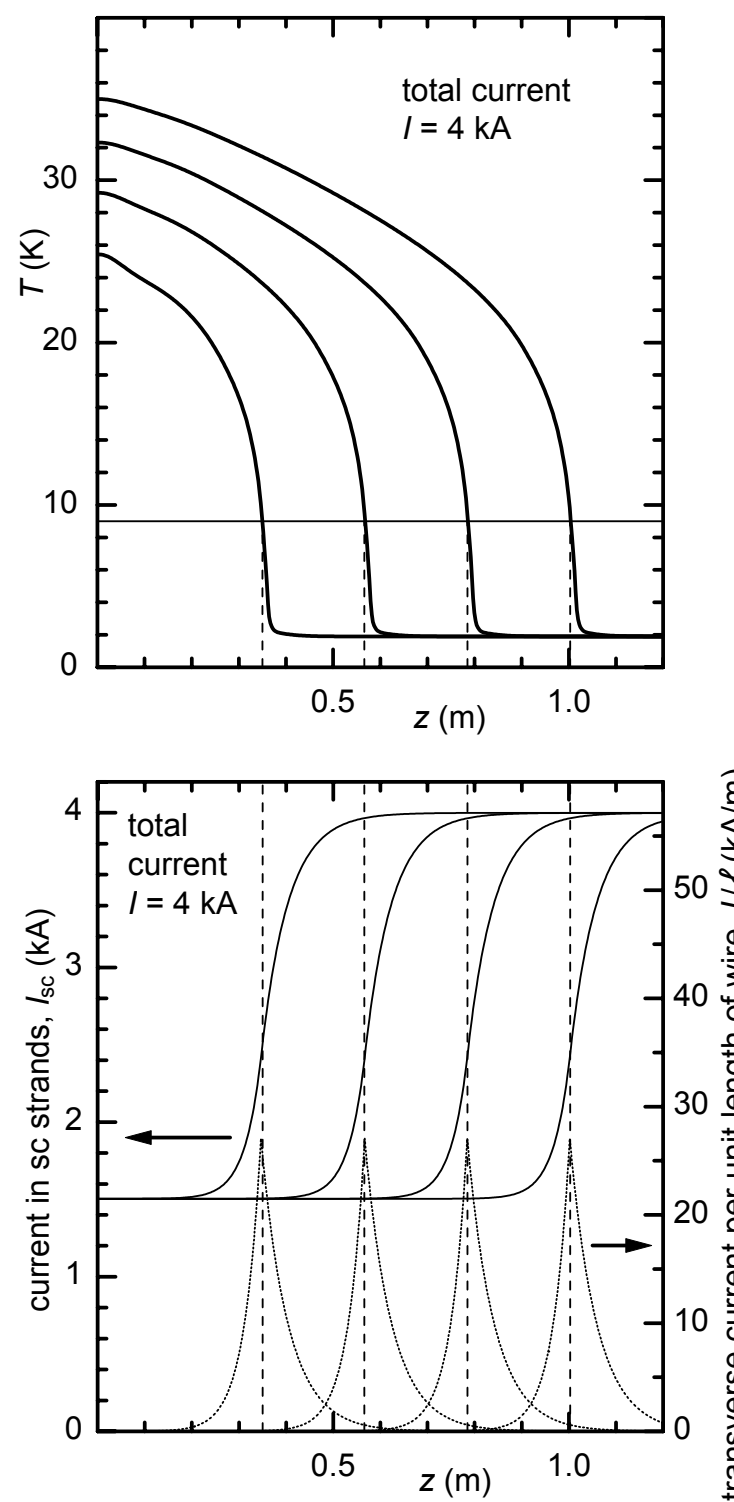

FIGURE 7. Simulation of temperature profile, current in the superconducting strands and transversal current at 4 time instances. The current crosses in a $30 \mathrm{~cm}$ long region from the normal to the sc. strands. rapidly. Between $t_{2}$ and $t_{3}, V_{1 / 2}$ rises nearly linearly; only an increase of the resistivity for $I>I_{1}$ leads to an additional voltage. At $t_{3}$, the quench passes tap 2, whose potential rises sharply causing a small reduction of $V_{1 / 2}$. The feature of the resulting peak, first observed in the experiments, stimulated the development of the model outlined here. It can not be seen in single strand conductors, because the two potentials of the superconducting and pure copper strands are averaged out to a curve without receding slope.

FIG 9 shows measured and simulated voltages between two adjacent taps at 2, 4 and $6 \mathrm{kA}$. The three simulations were calculated with the same values for the helium cross section, the contact resistance between superconducting and normal strands and all other geometric and materials parameters. Considering the simplicity of the model, the agreement between measurements and simulations is very good.

\section{PROTECTION OF CIRCUITS}

In a superconducting system with active quench protection, there are two distinct phases after the occurrence of a quench: the quench detection phase and the energy extraction phase. Usually a quench is detected by the resistive voltage of the circuit crossing a preset threshold value. After some delay caused by electronic filtering and other effects, energy extraction 


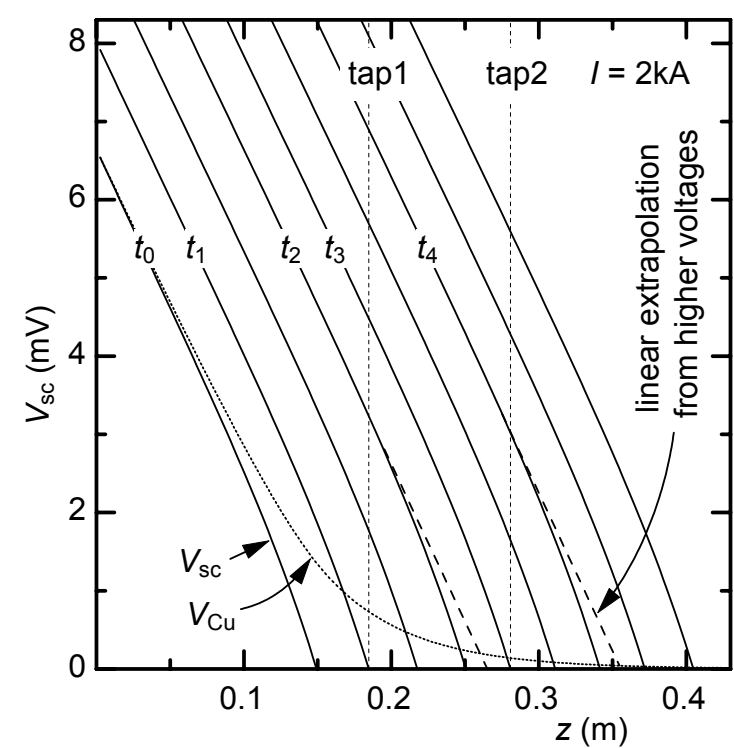

FIGURE 8a. Simulation of potentials in superconducting $\left(V_{\mathrm{sc}}\right)$ and pure copper strands $\left(V_{\mathrm{Cu}}\right)$ around the front line of an expanding normal zone at several time instances. $V_{\mathrm{Cu}}$ is unequal 0 well into the superconducting zone.

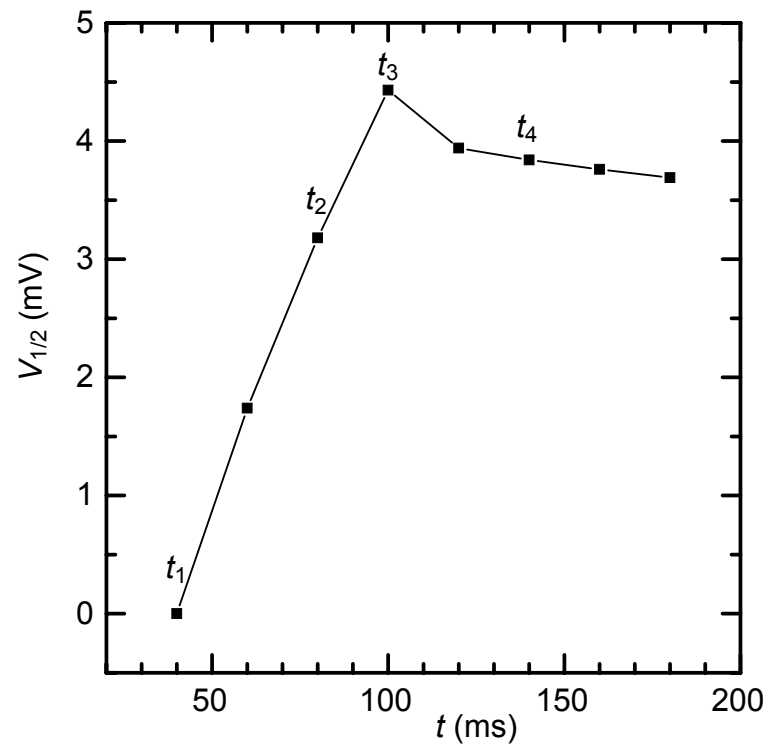

FIGURE 8b. The voltage (potential difference) between taps 1 and 2 . The times $t_{1}$ to $t_{4}$ correspond to the ones in FIG 8a.

starts. For the circuits containing the flexible $6 \mathrm{kA}$ cable, this is achieved by quenching the only magnet in the circuit with specially installed quench heaters. The resistance of this magnet then rises within $150 \mathrm{~ms}$ to large enough values to reduce the current in the circuit to zero.

In case a quench starts in the cable carrying a current of $6 \mathrm{kA}$, a typical detection voltage of $0.3 \mathrm{~V}$ is reached after $0.1 \mathrm{~s}$, when the normal zone reaches a length of $1.5 \mathrm{~m}$ and the hot-spot temperature exceeds $40 \mathrm{~K} .300 \mathrm{~ms}$ later the current is zero and $T_{\max }$ is $120 \mathrm{~K}$. Detection at high currents is easy, because the quench propagation velocity is high and a comparatively small resistance is sufficient to reach the threshold voltage.

At currents below $I_{0}(\sim 950 \mathrm{~A})$, a quench collapses and no reaction of the protection system is necessary. The system returns to its normal operating condition by itself.

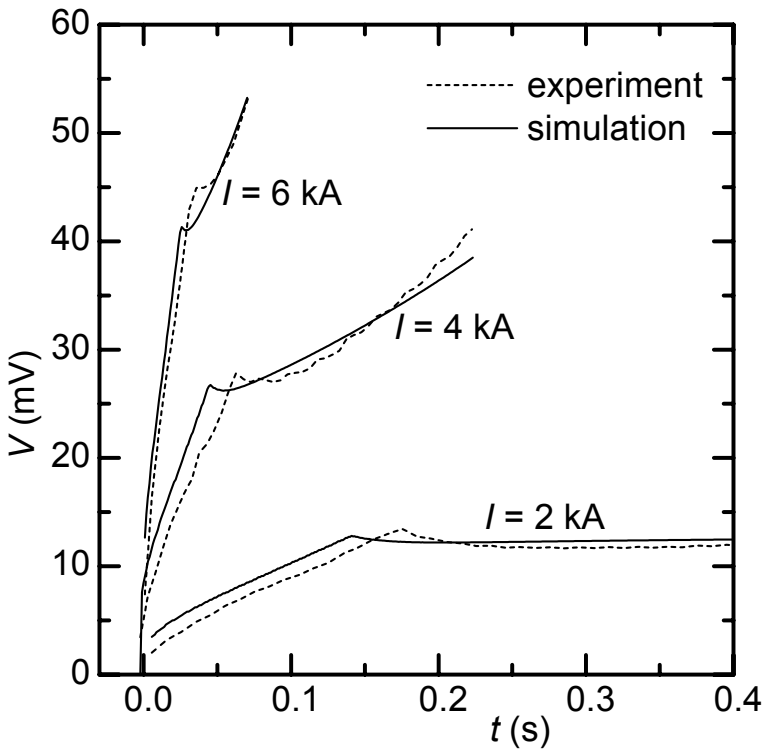

FIGURE 9. Measured and simulated voltage between two taps for normal zones expanding from one tap to the other at currents of 2,4 and $6 \mathrm{kA}$.
The most difficult case arises at intermediate currents, just above $I_{1}$ $(\sim 1500 \mathrm{~A})$, where a quench propagates slowly and the resistance of the normal zone needs to be large to reach the detection voltage. Unlike the 600 A busbars studied in [4], experiments showed that the equilibrium between cooling and heating in the normal state disappears after a few seconds in the $6 \mathrm{kA}$ conductors, most likely because the helium environment changes. In this situation, a number of simulations were conducted with various time dependent cooling scenarios in the attempt to find the worst condition, i.e. highest $T_{\max }$, after detection and subsequent energy extraction. The detection level used was $0.3 \mathrm{~V}$. It was found that $T_{\max }$ never exceeds 
$250 \mathrm{~K}$, even without cooling at the hot spot and good cooling $1 \mathrm{~m}$ away, if the normal zone is allowed to expand at least $6 \mathrm{~m}$ on either side of the hot spot (or $12 \mathrm{~m}$ on one side). From a quench protection point of view, it therefore seems safe to operate the prototype busbar at all currents between 0 and $6 \mathrm{kA}$.

\section{CONCLUSIONS AND IMPLICATIONS}

The use of superconducting and pure copper strands in parallel for a single conductor, rather than all superconducting strands, leads to an increase of the quench propagation velocity because heating occurs already 'ahead' of the front line of the normal zone, in a region with $T$ well below $T_{\mathrm{c}}$. In case of an expanding quench, it is important to create the voltage which triggers the quench detection system as soon as possible to start the energy extraction with the lowest possible hot-spot temperature. Here the larger quench propagation velocity helps, because the necessary resistance is created on a long and 'cold' normal zone, instead of a short and 'hot' one. It is apparent that a quench should not be stopped, which might happen at joints where the copper cross section is increased. The busbar layout should assure that at least no sections shorter than $12 \mathrm{~m}$ with such potential stoppers are foreseen.

The use of two strand types leads to a peak in the voltage between two taps when the front line of a normal zone passes the second tap. This feature is a consequence of the current which passes from one type of strand to the other and the associated potential in the two types. A simple model manages to reproduce the measured curves with good accuracy.

An open question for the use of the $6 \mathrm{kA}$ cable in the LHC, which has not been addressed yet, is the electrical compatibility of two or three such cables next to each other in a single tube. It is hoped that tests foreseen for the LHC String 2 installation will clarify this question. The operation of the cable in a transversal field of $1.5 \mathrm{~T}$ without the appearance of quenches is already encouraging.

\section{ACKNOWLEDGEMENTS}

We would like to thank Lev Vassiliev and Vladimir Sytchev from the Institute of High Energy Physics in Protvino and V.E. Sytnikov from VNIIKP in Podolsk, Russia for their help with the design and production of the prototype cable. Many thanks to the LHC/MTA team in Block 4 at CERN who installed the test assembly in the cryostat and generously offered all their expertise and measurement equipment. Finally we are happy to thank our colleague Knud Dahlerup-Petersen for his initiative to launch the cooperation with IHEP and his support in many ways for our project.

\section{REFERENCES}

1. Herzog, R., Dahlerup-Petersen, K., Parente, C., Schmidt, R., Sonnemann, F. and Teng, M., "The Superconducting Busbar Cable Powering the Auxiliary Magnets of the LHC" in Advances in Cryogenic Engineering 45B, edited by Q.S. Shu et al., Kluwer Academic / Plenum, New York, 2000, pp. 1581-1588.

2. Sonnemann, F. and Schmidt, R., Cryogenics 40, pp. 519-529 (2000).

3. Sytnikov, V.E., Svalov, G.G., Peshkov, I.B. Cryogenics 29, pp. 971-974 (1989).

4. Herzog, R., Calvi, M., Sonnemann, F., "Quench Propagation and Heating in the Superconducting 600 A Auxiliary Busbars of the LHC", see previous article in these proceedings.

5. Sonnemann F. and Schmidt R., Cryogenics 40, pp. 519-529 (2000). 